\title{
Experimental Investigations of Rotary Electromagnetic Mould Stirring in Continuous Casting Using a Cold Liquid Metal Model
}

\author{
Bernd WILLERS, ${ }^{1)}$ Martin BARNA, ${ }^{2)}$ Jürgen REITER ${ }^{3)}$ and Sven ECKERT ${ }^{1) *}$ \\ 1) Helmholtz-Zentrum Dresden-Rossendorf (HZDR), Institute of Fluid Dynamics, Bautzner Landstraße 400, 01328 Dresden, \\ Germany. 2) Johannes Kepler University (JKU) Linz, Institute of Fluid Mechanics and Heat Transfer (ISW), \\ Altenbergerstraße 69, 4040 Linz, Austria. $\quad 3)$ Voestalpine Stahl Donawitz GmbH, Kerpelystraße 199,8700 Leoben,
} Austria.

(Received on September 9, 2016; accepted on November 9, 2016; J-STAGE Advance published date: February 10, 2017)

\begin{abstract}
This paper presents the experimental study of an electromagnetically stirred mould flow using a 1:3 scale acrylic glass model of the round bloom caster from voestalpine Stahl Donawitz GmbH. An electromagnetic stirrer was installed at the strand producing a rotating magnetic field (RMF). Flow measurements were performed in the eutectic alloy GalnSn at room temperature by means of the ultrasound Doppler velocimetry (UDV). Up to 10 ultrasonic transducers were employed simultaneously in order to obtain a two-dimensional reconstruction of the flow structure. The experiments contribute to a better understanding of electromagnetically stirred mould flows and provide an extensive and valuable data base for the validation of numerical methods. The flow measurements reveal a distinct influence of the secondary flow on the distribution of the angular velocity in various regions of the mould. The submerged jet intensifies this secondary motion in the upper part of the mould and thus causes a strong deformation of the free surface of the melt. The jet is deflected, bent and rotates around the strand axis.
\end{abstract}

KEY WORDS: continuous round bloom casting; eutectic alloy GalnSn; experiments; electromagnetic stirring; rotating magnetic field; ultrasound Doppler flow measurements.

\section{Introduction}

Electromagnetic stirring (EMS) by means of AC magnetic fields is a valid method for contactless flow control in billet/bloom casters for about 40 years, ${ }^{1-4)}$ where the rotary stirring of the mould flow by means of a rotating magnetic field (RMF) can be considered as an established application. The stirring is supposed to enhance the homogeneity of the molten steel and to improve the quality of the solidified steel strand therefore significantly decreasing the number of casting defects. In particular, it is assumed that non-metallic particles are prevented from becoming entrapped in the solidified shell by the so-called washing effect. ${ }^{5)}$ Particles attached to the growing columnar dendrites are prevented from engulfment by the mushy zone, washed out by the forced flow and transported back into the bulk liquid. In addition, the stirring shall stimulate the agglomeration and the formation of larger particles which rise up more easily towards the free surface. Yamada et al $^{6}{ }^{6}$ analyzed the size of alumina inclusions in solidified steel. They suggested that the maximum diameter of the clusters remaining in the product corresponds to the EMS- induced velocity of molten steel. On the basis of these data Matsumiya ${ }^{7)}$ concluded that inclusions with a size of $100 \mu \mathrm{m}$ can be prevented from

\footnotetext{
* Corresponding author: E-mail: s.eckert@hzdr.de

DOI: http://dx.doi.org/10.2355/isijinternational.ISIJINT-2016-495
}

embedding into the solidified shell by flow velocities of about $0.3 \mathrm{~m} / \mathrm{s}$. Using an experimental model in which molten steel was stirred by an RMF during solidification Miki et al. ${ }^{8)}$ observed an enrichment of agglomerate inclusions at the axis of rotation in the resulting ingots.

Moreover, it is expected that the forcing of melt flow in close vicinity to the solidification region promotes a transition from columnar to equiaxed solidification. An intense flow along the solidification front causes a fragmentation of the dendrites and hence a multiplication of nuclei. Su et al. ${ }^{9)}$ suggest a dendrite fragmentation criterion for low carbon and high carbon steels casted under the influence of EMS.

Industrial experience has shown that the particular stirrer design, position and operating conditions have a strong influence on the metallurgical quality. First of all, a high stirring intensity should be guaranteed by a suitable coil design and appropriate magnetic field parameters. A stirrer operation at low magnetic field frequencies (typically 2 to $8 \mathrm{~Hz}$ ) is required because at higher frequencies the thick copper walls of the mould impede a deep penetration by the AC magnetic field. This restriction to low frequencies limits the driving electromagnetic Lorentz force. Furthermore, an intensive swirling flow at the free surface poses the risk of slag or mould powder entrapment due to the depression of the surface around the nozzle. The absorption of impurities is known to lower the quality of steel products significantly. A specific electromagnetic stirring system of two stirrers 
was considered to influence the meniscus stability. ${ }^{10)}$ Two independent rotating magnetic fields superimposed upon each other are applied to achieve a flexible control of the stirring motion in the meniscus zone regardless of whether an intensive stirring is generated deeper in the mould. In this way even an opposite stirring direction near meniscus can be chosen with respect to the main stirrer.

A considerable amount of previous research work was dedicated to the prediction of the mould flow and related heat and mass transfer under the action of EMS, mainly by numerical simulations (see for example). ${ }^{11-17)}$ Numerical calculations can provide a better understanding of the complex flow behaviour, but experimental data are indispensable with respect to validation of these CFD models. Experimental investigations in an electromagnetically stirred Woods metal model were performed by Partinen et al. ${ }^{12,18)}$ The authors measured the deformation of the free surface of the melt and determined the surface velocity using alumina particles on the surface. The motion of these particles was recorded by means of a high-speed video camera. Fluid flow measurements within the liquid metal were carried out using an electromagnetic Vives probe. The comparison between the measurements and the results obtained by the numerical model shows fairly good agreement, but the measurements of the bulk flow by the Vives probe turned out to be rather challenging. The authors reported severe problems arising from the size of the invasive probe and the interference of the magnetic field driving the flow and the field of the permanent magnet incorporated in the probe. In particular, it became apparent that the immersion of the probe into the melt caused distinct changes of the surface deformation. Since this pioneering work performed 20 years ago new ultrasonic measuring techniques were adapted to be applied successfully in liquid metal flows. ${ }^{19-21)}$ The used ultrasonic Doppler velocimetry (UDV) is a non-intrusive method which instantaneously delivers the liquid velocity profile along the ultrasonic beam. New developments of ultrasonic sensor arrays allow for a multidimensional flow mapping. ${ }^{22)}$ Such imaging techniques become more and more important for detailed explorations of three-dimensional turbulent flows, in particular with respect to the generation of a suitable experimental data base for an efficient validation of numerical simulations.

The motivation of our study is to provide a rather generic experiment equipped with advanced ultrasonic flow measuring technique for detailed investigations of the liquid metal flow in a long cylindrical column driven by an RMF. Especially, the consequences of variations of the field strength and the frequency on the stirring intensity and the behavior of the free surface are considered here. Furthermore, we compare the case of a pure RMF-driven flow with the more realistic configuration where the swirling flow in the mould is superimposed by an emergent jet from a vertically aligned submerged entry nozzle. The flow measurements were conducted in a 1:3 scale acrylic glass model of the round bloom strand caster from voestalpine Stahl Donawitz GmbH. A description of the model experiment, the magnetic field system and the measuring technique will be given in the subsequent chapter. The measured results are presented and discussed in the chapters 3 and 4, respectively. Concluding remarks can be found in chapter 5 .

\section{Experimental Setup}

\section{A - The flow model}

The experiments were conducted at the mini-LIMMCAST facility at HZDR. A detailed description of this experimental equipment can be found in previous publications. ${ }^{23,24)}$ About 12 litres of the ternary eutectic alloy $\mathrm{Ga}_{68} \mathrm{In}_{20} \mathrm{Sn}_{12}$, which is liquid at room temperature, were used as a model fluid. ${ }^{25)} \mathrm{A}$ schematic view of the experimental facility can be seen in Fig. 1. The tundish is a cylindrical vessel made of stainless steel with a circular outlet into the submerged entry nozzle (SEN). A stopper rod controls the mass flow rate through the SEN. The SEN is an ordinary circular pipe made of acrylic glass with an inner diameter of $10 \mathrm{~mm}$ and a length of $300 \mathrm{~mm}$. A SEN immersion depth of $35 \mathrm{~mm}$ below the surface level of the liquid metal is chosen for the experiments presented here. The mould is also made of acrylic glass and has a circular cross section with an inner diameter of $80 \mathrm{~mm}$ and a length of $800 \mathrm{~mm}$. The melt discharges from the SEN into the mould as a submerged jet. To close the experimental test loop, after the mould the liquid metal flows over a dam into a storage vessel. The vertical position of the spillway controls the free surface level in the mould. The experiments presented here were performed in a continuous mode. A magnetic pump conveys the melt from the vessel into the tundish at a maximum rate of about $7.5 \mathrm{l} / \mathrm{min}$. Owing to the eddy currents the operation of the pump causes a temperature rise in the metallic melt of approx. $0.1 \mathrm{~K} / \mathrm{min}$. The liquid flow rate was controlled by lifting the stopper rod into a defined position. The liquid levels in both the tundish and the storage vessel were monitored using a laser distance sensor. The tundish level was controlled and kept constant during the continuous operation by readjusting the pumping rate. Maximum level fluctuations of $\pm 10 \mathrm{~mm}$ were observed in a period of about two hours. The flow measurements were started after achieving fully developed steady flow conditions. Flow rate measurements were conducted

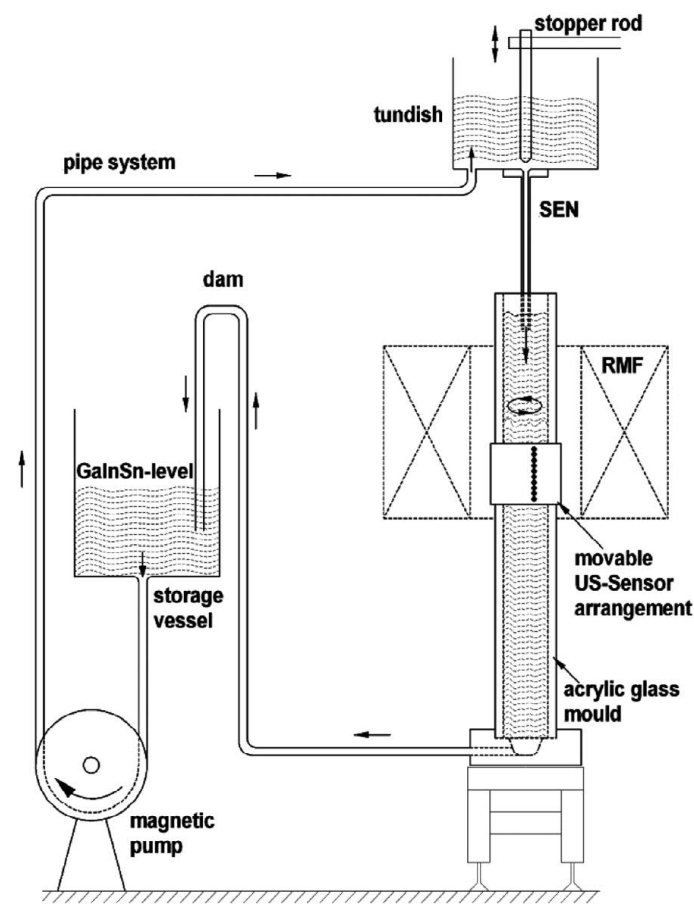

Fig. 1. Schematic drawing of the experimental setup. 
at the end of each measuring campaign when the pump was switched off. While emptying the tundish the overall liquid flow rate can be derived from the descent of the surface level in the tundish. The velocity inside the SEN could be estimated in this indirect way to be in the range of $1.2-1.5 \mathrm{~m} / \mathrm{s}$.

\section{$B$ - The magnetic field system}

The electromagnetic system has a bore diameter of $200 \mathrm{~mm}$ and a height of $310 \mathrm{~mm}$. An arrangement of 12 coils generates a rotating magnetic field (RMF) with a maximum magnetic flux density of $\mathrm{B}=20 \mathrm{mT}$. The coils are installed around the mould with the top edge of the magnetic stirrer located $60 \mathrm{~mm}$ below the free surface of the liquid metal. Systematic flow measurements were conducted for different values of the magnetic field strength (peak value) varying in the range between $4.1 \mathrm{mT}$ and $18.3 \mathrm{mT}$. Magnetic field frequencies between $2.5 \mathrm{~Hz}$ and $50 \mathrm{~Hz}$ were chosen. Every change of the magnetic field parameters in the course of a measuring campaign was accompanied by an idle time of at least 2 min prior to the next measurement. This was necessary in order to guarantee a complete adaptation of the flow pattern to the new magnetic field parameters. A Gauss meter (LakeShore model 460, sensor type MZZ-2512-UH) was applied to determine the field distribution. The spatial distribution of the magnetic flux density measured for a feeding current $\mathrm{I}=10 \mathrm{~A}$ and a field frequency $\mathrm{f}=10 \mathrm{~Hz}$ is presented in Fig. 2(a). Figure 2(b) shows the vertical profile of the magnetic field measured along the axis for three different combinations of field strength and stirring frequency. A domain of uniform field strength occurs in the inner zone of the stirrer whereas the magnetic induction starts to decrease at a vertical distance of about $50 \mathrm{~mm}$ from the edges of the stirrer. The magnetic system generates a remarkable stray field, which extends to mould regions far below the stirrer.

\section{$C$ - Flow measurement technique}

The fluid velocity in the mould was measured by means of the ultrasound Doppler velocimetry (UDV). This method is based on the pulse-echo technique and delivers instantaneous profiles of the velocity component projected onto the propagation direction of the ultrasonic beam. ${ }^{19)}$ The measurements in the present study were performed using the DOP2000 velocimeter (model 2125, Signal Processing SA, 1073 Savigny, Switzerland). This instrument is equipped with an internal multiplexer allowing for sequential data acquisition from up to ten sensors.

The outer cylindrical wall of the mould was partly machined to a flat surface in order to enable an exact positioning of the US sensors at different distances to the strand axis and heights. The transducers were attached at the outer wall using a technical vaseline. Acrylic glass and GaInSn are a well-suited material combination for non-invasive ultrasound measurements through the mould wall because these two materials have almost the same sound velocity of

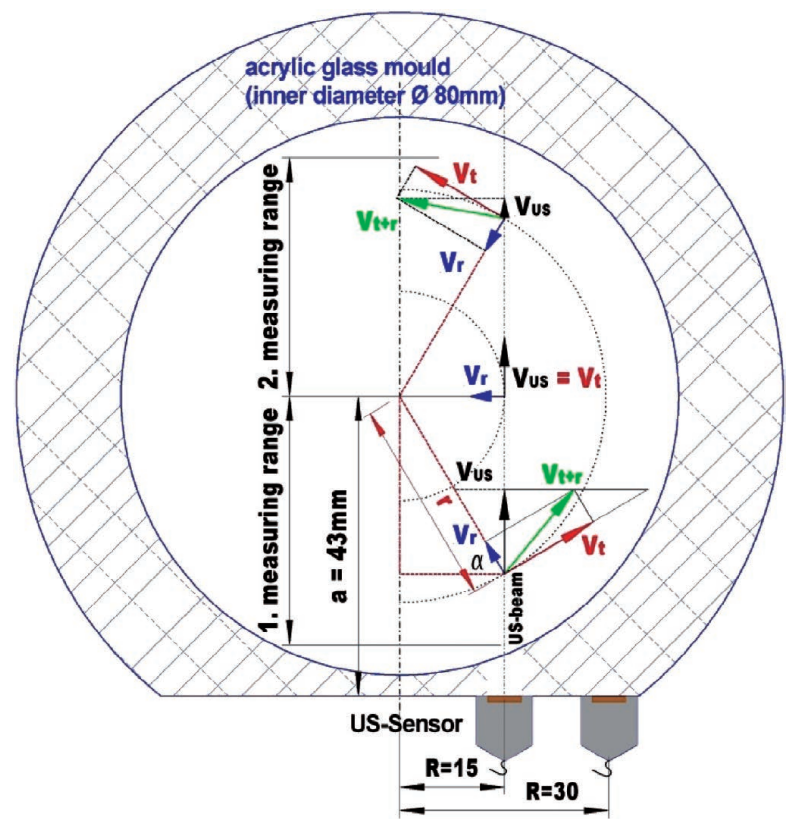

Fig. 3. The schematic view showing the off-center measuring positions of the US Sensors with US-beam lines at $\mathrm{R}=15$ $\mathrm{mm}$ and $\mathrm{R}=30 \mathrm{~mm}$ and the horizontal velocity vectors of tangential and radial flow component.
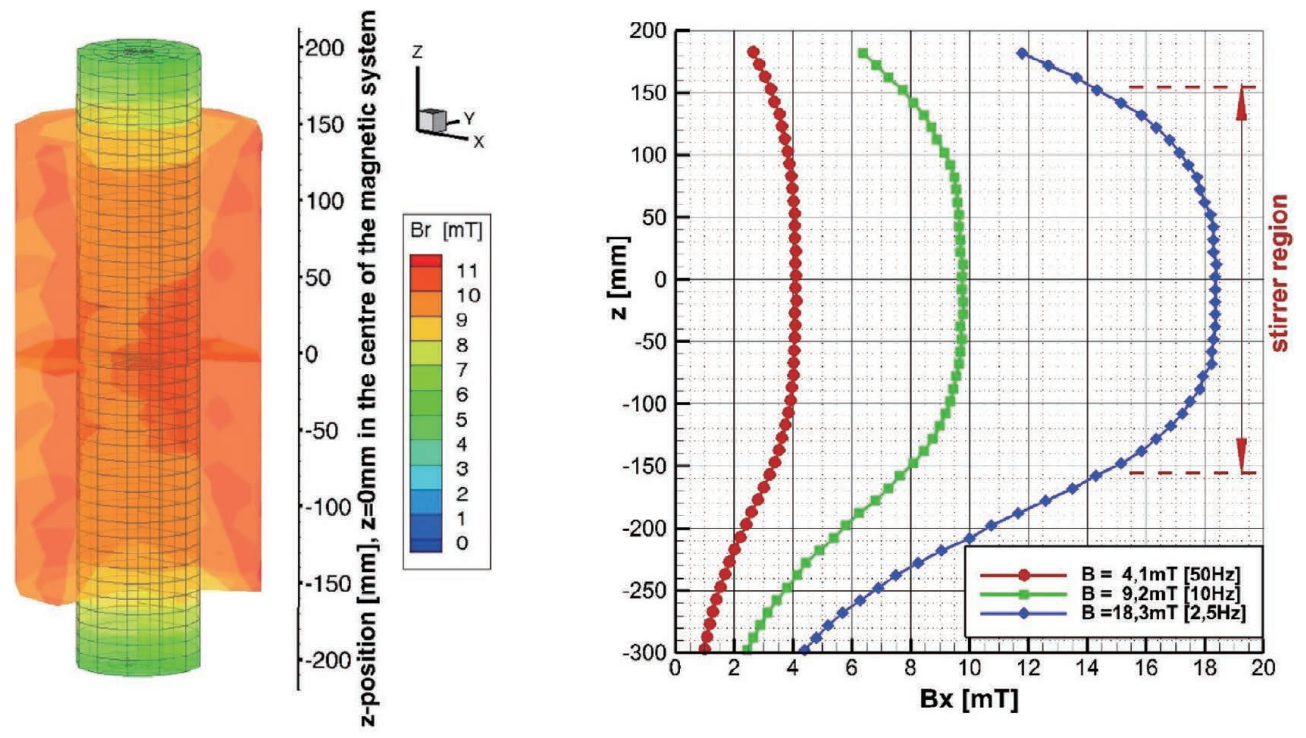

Fig. 2. (a) Magnetic flux density inside the RMF; the mesh represents a volume of $\varnothing 80 \times 410 \mathrm{~mm}^{2} ; \mathrm{I}=10 \mathrm{~A}, \mathrm{f}=10 \mathrm{~Hz}$; $\mathrm{B}=9,9 \mathrm{mT}$ in the center of the RMF. (b) Magnetic flux densities at the centerline inside and outside of the stirrer region. 
approximately $c_{s}=2750 \mathrm{~m} / \mathrm{s}{ }^{26)}$ Hence, velocity measurements through the wall are possible as there is no significant diffraction of sound at the solid-liquid phase boundary.

A vertical array of ten $4 \mathrm{MHz}$ transducers (TR0405AS, acoustic active diameter $5 \mathrm{~mm}$ ) was assembled with a distance of $10 \mathrm{~mm}$ between two adjacent transducers. Horizontal velocities were measured along chords parallel to the diameter of the strand situated at specific distances from the axis of the cylindrical mould, namely at the positions $\mathrm{R}=15 \mathrm{~mm}$ and $\mathrm{R}=30 \mathrm{~mm}$. The locations of the measuring lines in the mould cross section are displayed in Fig. 3. The exact values of the tangential velocity can be detected on both radial measuring positions at a distance of $\mathrm{a}=43 \mathrm{~mm}$ from the sensor where the propagation line of the ultrasonic beam is parallel to the tangential direction of the flow velocity.

The height of the model facilitates a vertical measuring range of $660 \mathrm{~mm}$. The centre of the electromagnetic stirrer was taken as the vertical zero point of the coordinate system. The vertical distance of $10 \mathrm{~mm}$ between neighbouring sensors leads to $2 \times 67$ fixed acoustic beam lines for acquiring linear velocity profiles. The data recording for the multiple sensor arrangement was conducted by multiplexing using an overall scan rate between $3 \mathrm{~Hz}$ and $10 \mathrm{~Hz}$ for all 10 ultrasonic sensors. The measuring volume of a particular sensor comprises a sequence of separate cylindrical disks lined up concentrically along the propagating ultrasound beam. In the present study, the size of the individual disks in the axial direction was about $1 \mathrm{~mm}$. Owing to the divergence of the ultrasonic beam the lateral size of the disks increases with increasing distance from the transducer. The divergence angle of the $4 \mathrm{MHz}$ transducers used in this study is $\varphi=$ $2.3^{\circ}{ }^{26)}$ Hence the lateral resolution varies from $5 \mathrm{~mm}$ at the sensor to approximately $9 \mathrm{~mm}$ at a distance of $100 \mathrm{~mm}$ from the sensor. The velocity resolution is about $0.4 \%$ of the velocity range, i.e. a resolution of $4.4 \mathrm{~mm} / \mathrm{s}$ can be achieved for a velocity range of $1.1 \mathrm{~m} / \mathrm{s}$.

\section{Experimental Results}

\subsection{Rotating Flow without Submerged Jet}

A first campaign of velocity measurements was carried out for the situation of a solely rotating flow without the influence of a submerged jet.

Vertical profiles of the mean tangential velocity $\mathrm{V}_{\mathrm{t}, \mathrm{r} 30}$ obtained at $\mathrm{R}=30 \mathrm{~mm}$ for six different magnetic field intensities and stirring frequencies are shown in Fig. 4(a). The time-averaged values are calculated from 100 to 300 flow profiles recorded over a period of ca. $30 \mathrm{~s}$ to $60 \mathrm{~s}$. The magnetic stirrer was positioned in the domain highlighted by the two horizontal black lines in the diagram. The flow is driven by the Lorentz force which is mainly induced inside the stirrer region. Hence, the largest values of the mean tangential velocity approximately coincide with the geometric centre of the stirrer. Internal friction and secondary flows are responsible for the rotating motion of the fluid above and below the magnetic stirrer. At a vertical position of $\mathrm{z}=-400 \mathrm{~mm}$ (245 $\mathrm{mm}$ beneath the stirrer's lower edge) a reduction of the tangential velocity to approximately $50 \ldots 60 \%$ of the maximum value is observed. The intensity of the swirling flow grows with increasing both

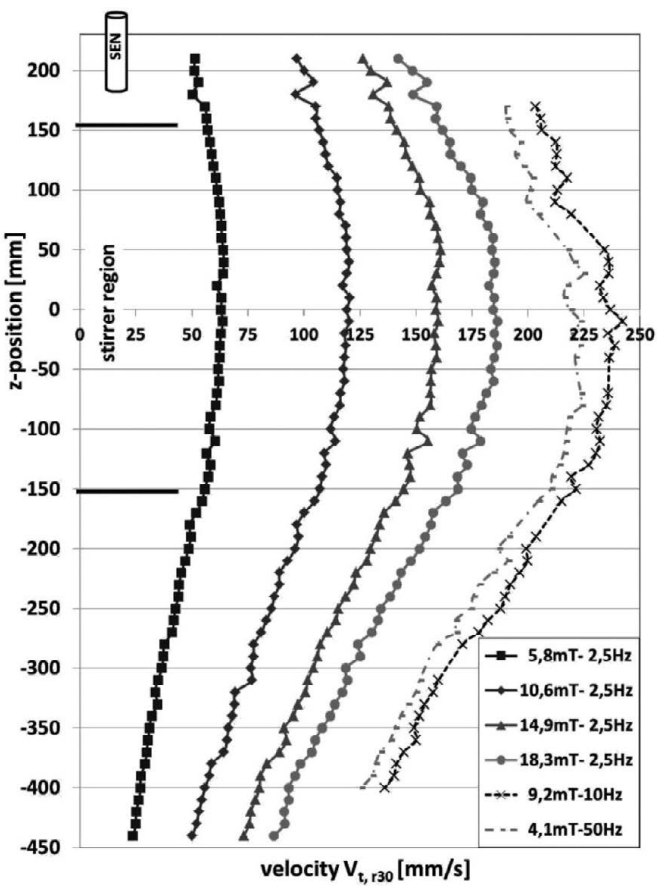

(a)

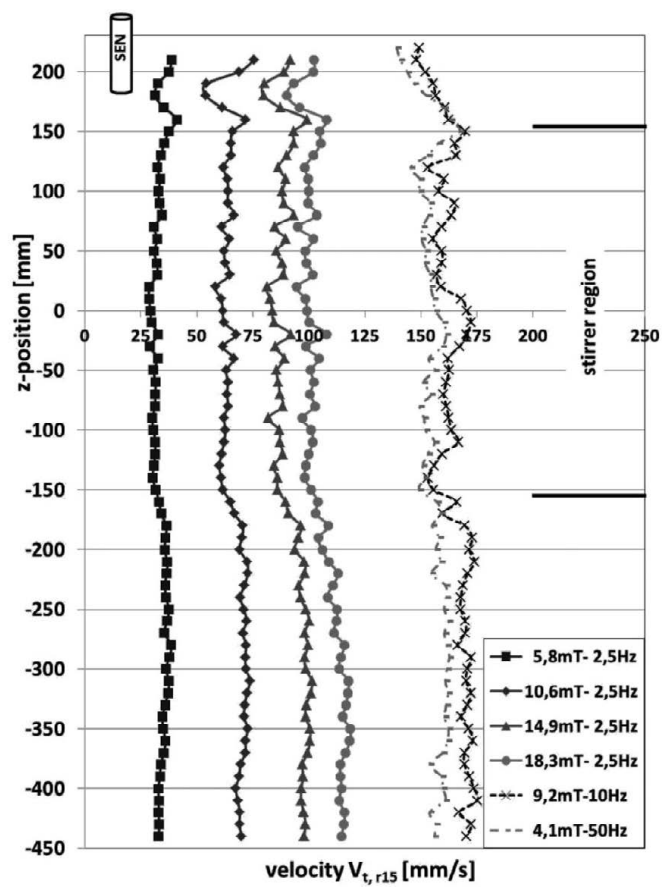

Fig. 4. Magnetic stirred rotating flow without the influence of a submerged jet. Vertical profiles of the time-averaged, tangential velocity measured at a radial position of $30 \mathrm{~mm}$ (a) Vertical profiles of the time-averaged, tangential velocity measured at a radial position of $15 \mathrm{~mm}$ (b).

field strength and frequency, for instance, the application of a magnetic field of $\mathrm{B}=18.3 \mathrm{mT}$ at $\mathrm{f}=2.5 \mathrm{~Hz}$ results in a maximum velocity of $\mathrm{V}_{\mathrm{t}, \mathrm{r}=30}=186 \mathrm{~mm} / \mathrm{s}$. The application of higher frequencies leads to higher fluid velocities, even at lower magnetic field intensities. However, because of the copper mould's shielding effect, it is quite inefficient to apply field frequencies of $10 \mathrm{~Hz}$ and larger at a real caster.

Figure 4(b) shows corresponding measurements of the mean tangential velocities recorded at the radial position $\mathrm{R}=$ $15 \mathrm{~mm}$. Although the tangential velocity is smaller at the lower radial position, the respective frequency of fluid rotation is almost the same $\left(\mathrm{f}_{\mathrm{F}, \mathrm{r}=15}=1.1 \mathrm{~s}^{-1}\right.$ and $\mathrm{f}_{\mathrm{F}, \mathrm{r}=30}=1 \mathrm{~s}^{-1}$ for a magnetic field frequency $f=2.5 \mathrm{~Hz}$ ). A remarkable 
difference with respect to the velocity profiles presented in Fig. 4(a) is the fact that the vertical velocity profiles do not show a pronounced maximum in the region of the magnetic stirrer. Instead, the tangential velocity in Fig. 4(b) appears to be almost uniform along the height of the strand showing only minor variations. The velocity in the lower part of the mould is even slightly exceeding the values measured within the stirrer region of the magnetic field. Although no jet flow was applied, the SEN was immersed into the melt during all measurements carried out at $\mathrm{f}=2.5 \mathrm{~Hz}$. The presence of the nozzle leads to a local braking of the flow which becomes apparent by undershoot in the velocity profiles in the domain $150 \mathrm{~mm}<\mathrm{z}<200 \mathrm{~mm}$. This drop in the tangential velocity can also be observed at an outer radial position (see Fig. 4(a)), however, the perturbation is not that pronounced here as in the region close to the cylinder axis.

As a next step we want to compile a horizontal profile of the tangential velocity along the ultrasonic beam line. In general, the measured horizontal velocity has to be decomposed into a tangential and a radial component as shown in Fig. 3 for the measuring line at $\mathrm{R}=15 \mathrm{~mm}$. Let us assume an axisymmetric flow, which basically means that the absolute value of all velocity components remains unchanged along the circumference at a given radius $r$. In the case of a radially inwards flow (converging flow) the radial and tangential velocity components $\mathrm{V}_{\mathrm{r}}$ and $\mathrm{V}_{\mathrm{t}}$, respectively, can be determined from the values $\mathrm{V}_{\mathrm{US}}$ measured by the UDV system by solving the following equations:

$$
\begin{gathered}
V_{U S}\left(\mathrm{a}_{1}\right)=V_{t} \cos \alpha+V_{r} \sin \alpha\left(\mathrm{a}_{1}<43 \mathrm{~mm}, 1 \text {. measuring range }\right) \\
V_{U S}\left(\mathrm{a}_{2}\right)=V_{t} \cos \alpha-V_{r} \sin \alpha\left(\mathrm{a}_{2}>43 \mathrm{~mm}, 2 \text {. measuring range }\right) \\
\text { whereas } \cos \alpha=R / r
\end{gathered}
$$

From values of the tangential velocity component $\mathrm{V}_{\mathrm{t}}$, the angular velocity $\omega$ can be easily derived as $\omega=\mathrm{V}_{\mathrm{t}} / \mathrm{r}$. Figure 5 shows radial profiles of the time-averaged angular velocity recorded at different heights $\mathrm{z}$ for different values of the magnetic field strength and frequency. Figure 5(a) contains the case of a magnetic field of $\mathrm{B}=18.3 \mathrm{mT}$ and a stirrer frequency of $2.5 \mathrm{~Hz}$. The diagram reveals flat profiles of the angular velocity near the core of the cylinder for positions inside and in the vicinity of the stirrer. This indicates the occurrence of a solid body rotation in a wide domain of the liquid metal column. In the lower part of the mould $(\mathrm{z} \leq$ $-200 \mathrm{~mm}$ ) the angular velocity increases with decreasing distance to the cylinder axis. That implies a remarkably higher rotation rate of the fluid in the core domain as in the outer zone. It is interesting to note that the core rotation rate below the stirrer is found to be larger than inside the stirrer where the fluid experiences the electromagnetic driving force directly. Corresponding results are presented in the Fig. 5(b) for a stirrer frequency of $10 \mathrm{~Hz}$ at $\mathrm{B}=9.2$ $\mathrm{mT}$ and in Fig. 5(c) for a stirrer frequency of $50 \mathrm{~Hz}$ at $\mathrm{B}=$ $4,1 \mathrm{mT}$, respectively. The shape of the curves appears to be similar as found for the low magnetic field frequency (see Fig. 5(a)), whereas the rotation rate inside the stirrer region decreases slightly with increasing distance from the mould axis at the higher magnetic field frequencies.

\subsection{Rotating Flow with Submerged Jet}

All results presented so far considered a rotating flow

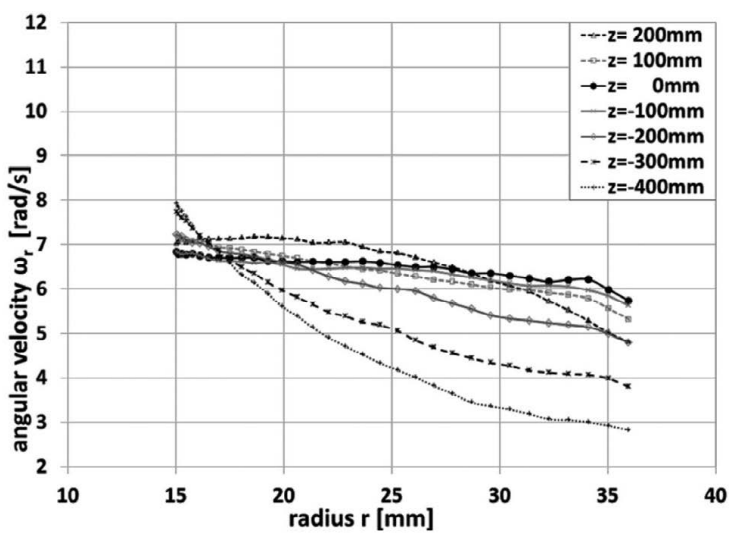

(a)

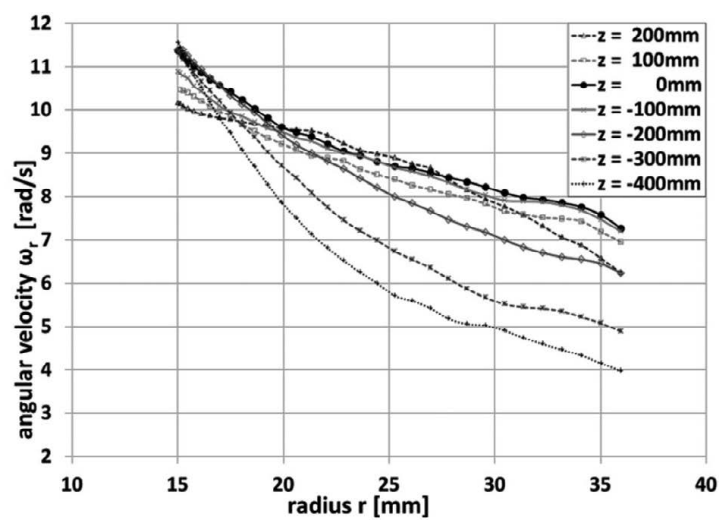

(b)

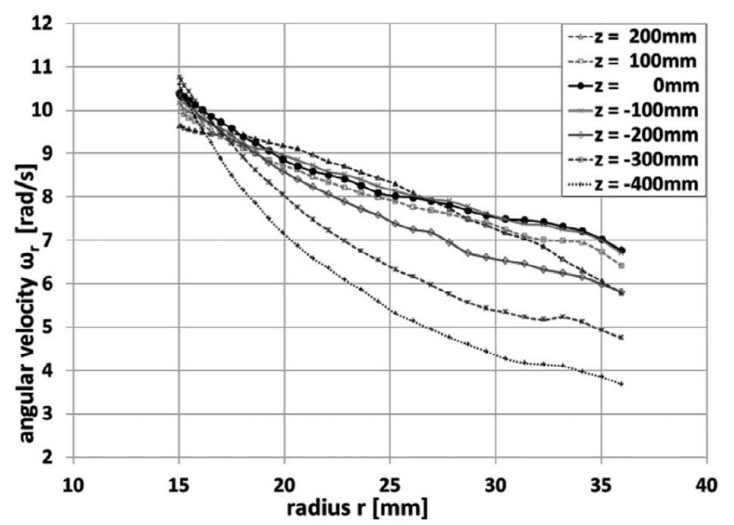

Fig. 5. Radial profiles of the angular velocity recorded along a chord at a distance of $\mathrm{R}=15 \mathrm{~mm}$ from the axis of the cylindrical fluid vessel in the first measuring range $(\mathrm{a}<43$ mm): (a) $\mathrm{B}=18.3 \mathrm{mT}, \mathrm{f}=2.5 \mathrm{~Hz}$, (b) $\mathrm{B}=9.2 \mathrm{mT}, \mathrm{f}=10$ $\mathrm{Hz}$, (c) $\mathrm{B}=4.1 \mathrm{mT}, \mathrm{f}=50 \mathrm{~Hz}$.

without the influence of a jet discharging from the SEN. The flow measurements in this section are concerned with the combination of the RMF-driven flow and the submerged jet. The behaviour of the tangential velocity was determined along the height of the cylindrical mould. Figure $\mathbf{6}$ displays the time-averaged velocity as well as the related minimum and maximum values obtained for a magnetic flux density of $18.3 \mathrm{mT}$ and a frequency of $2.5 \mathrm{~Hz}$ at radial positions of $30 \mathrm{~mm}$ (Fig. 6(a)) and $15 \mathrm{~mm}$ (Fig. 6(b)), respectively. For comparison the diagrams also contain the corresponding vertical profiles for the solely RMF-driven flow. The most striking difference with respect to the solely swirling flow is the fact that the fluid rotation rate is rather high near the free surface and reaches a distinct maximum just above the stirrer region. Moreover, it has to be noted that significantly higher tangential velocities were found at $r=15 \mathrm{~mm}$ in comparison to the measurements conducted at the position 
$\mathrm{r}=30 \mathrm{~mm}$. The corresponding curves in Fig. 6(b) reveal a significant drop of the tangential velocity in the upper part of the stirrer. The lowest values for the axial profile of $V_{t}$ along the entire mould length can be observed at a vertical position of $z=50 \mathrm{~mm}$. The spreading of the curves for the minimum and maximum values increases dramatically for the rotating flow with a superimposed jet, which indicates a much higher level of turbulent fluctuations than for the solely RMF-driven flow.

Figure 7 shows the vertical profiles of the time-averaged tangential velocity for the low stirring frequency of $2.5 \mathrm{~Hz}$ and different magnetic field intensities as well as for the

(a)
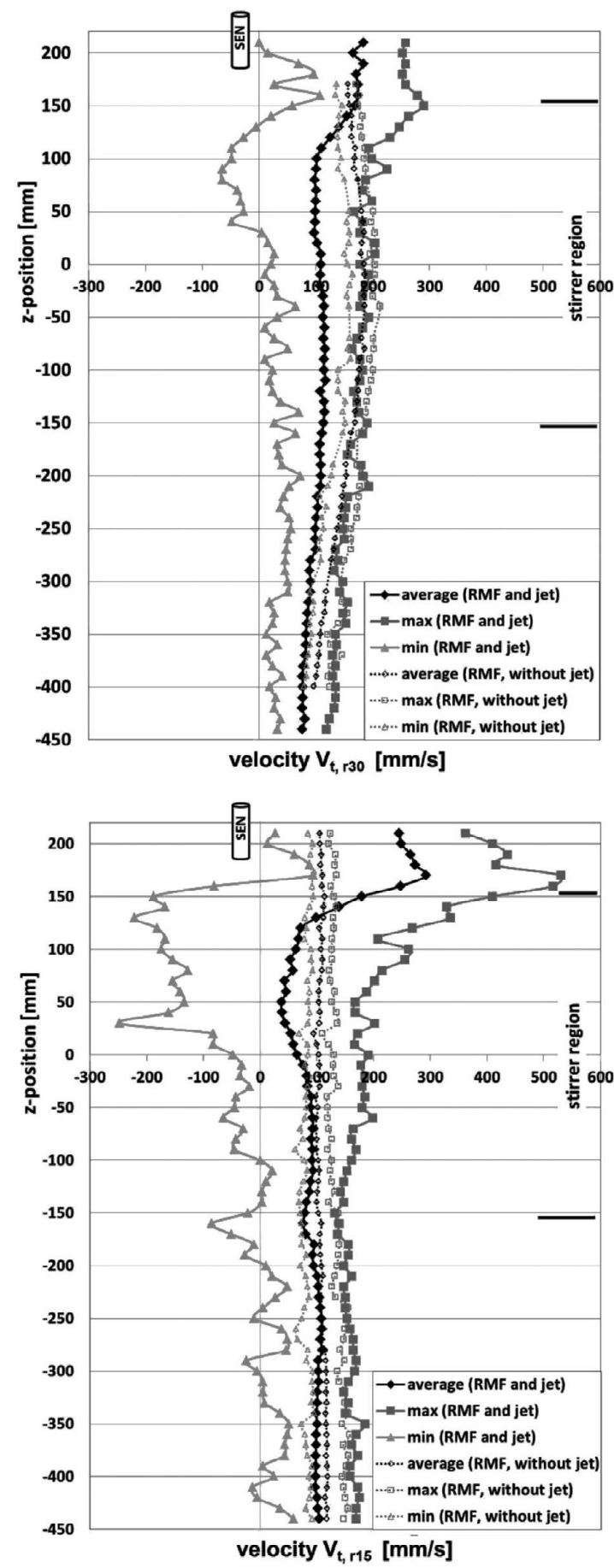

Fig. 6. Vertical profiles of the time-averaged, tangential velocity and both the corresponding minimum and maximum values measured at $\mathrm{B}=18.3 \mathrm{mT}$ and $\mathrm{f}=2.5 \mathrm{~Hz}$ at radial positions of $30 \mathrm{~mm}$ (a) and $15 \mathrm{~mm}$ (b), respectively. situation of a submerged jet without any stirring. No significant azimuthal flow can be found for the situation of the submerged jet without electromagnetic stirring. The graphs obtained for electromagnetic stirring and submerged jet show the maximum tangential velocity in the domain above the stirrer and the minimum inside the stirrer, whereas the velocity magnitude grows gradually with increasing magnetic flux density. It is interesting to note that an inversion of the velocity appears for the lowest field strength of $5.8 \mathrm{mT}$. The time average of the velocity reveals the occurrence of a domain ( $-30 \mathrm{~mm}<\mathrm{z}<60 \mathrm{~mm}$ ) with counter-rotating fluid (Fig. 7(a)). However, the existence of a flow pattern marked by a really counter-rotating flow in some regions is highly unlikely. It is rather likely that the interaction between the

(a)
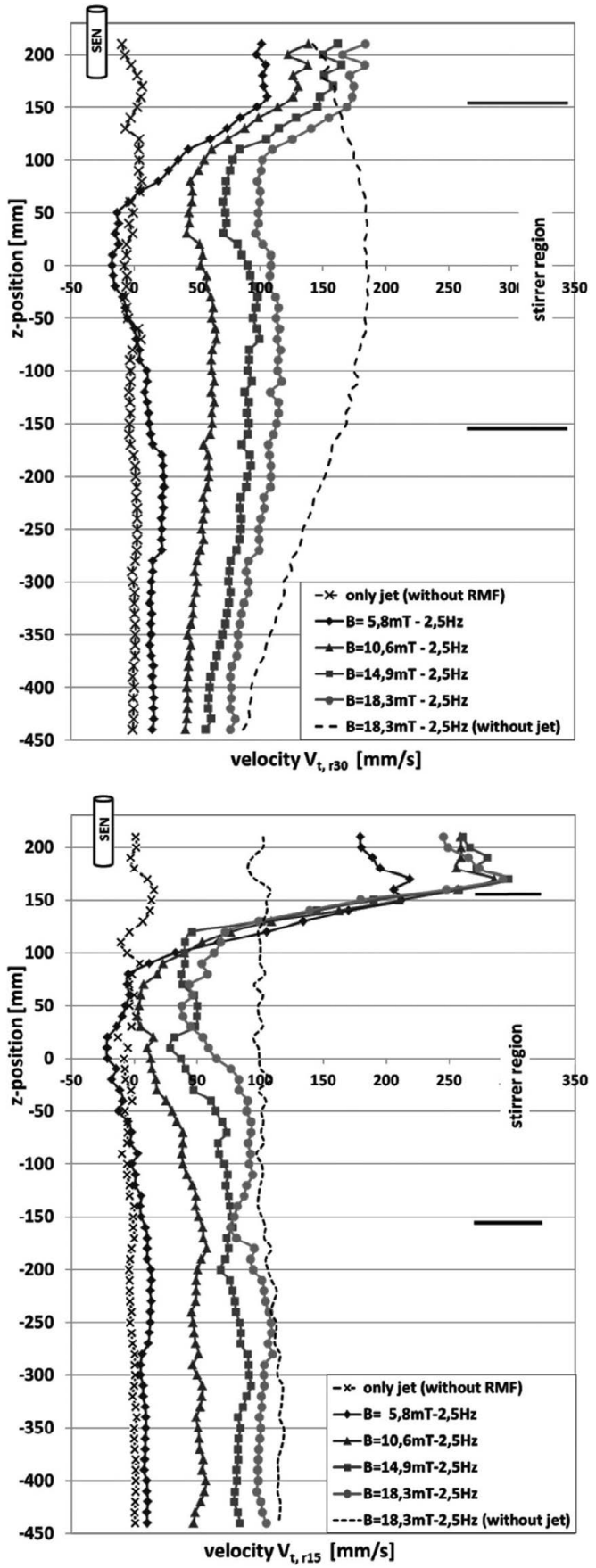

Fig. 7. Vertical profiles of the time-averaged, tangential velocity measured at radial positions of $30 \mathrm{~mm}$ (a) and $15 \mathrm{~mm}$ (b), respectively (various values of the field strength, $\mathrm{f}=2.5$ $\mathrm{Hz}$ ). 
jet and the rotating flow is responsible for the occurrence of this phenomenon.

In the next step the temporal behaviour of the rotating flow will be considered here. Figure 8 presents two-dimensional plots of the tangential velocity field obtained by a flow mapping using 10 ultrasonic transducers in a multiplex mode. The ultrasonic sensors were installed at $\mathrm{R}=15 \mathrm{~mm}$ along a vertical line at heights between $\mathrm{z}=130 \mathrm{~mm}$ and $\mathrm{z}=220 \mathrm{~mm}$

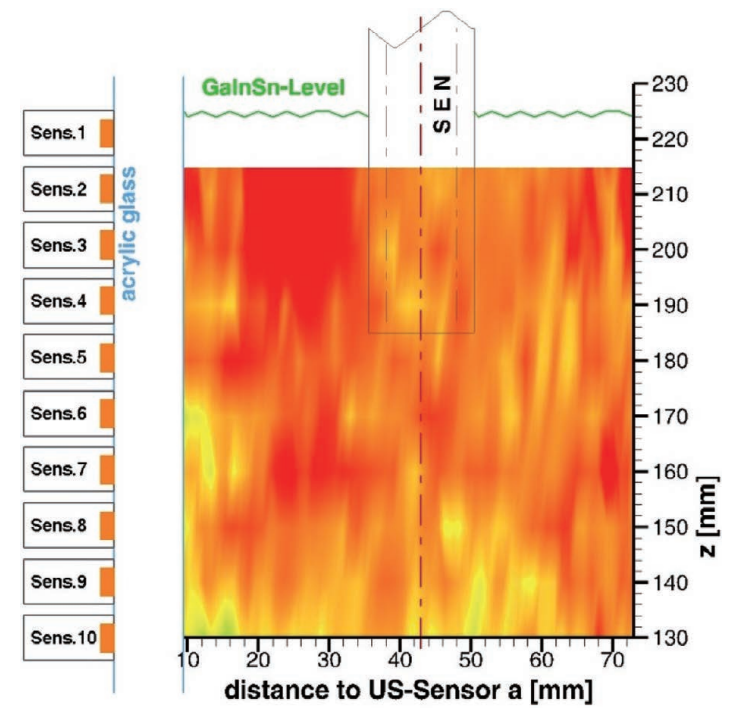

(a) $\mathrm{t}=0,0 \mathrm{~s}$

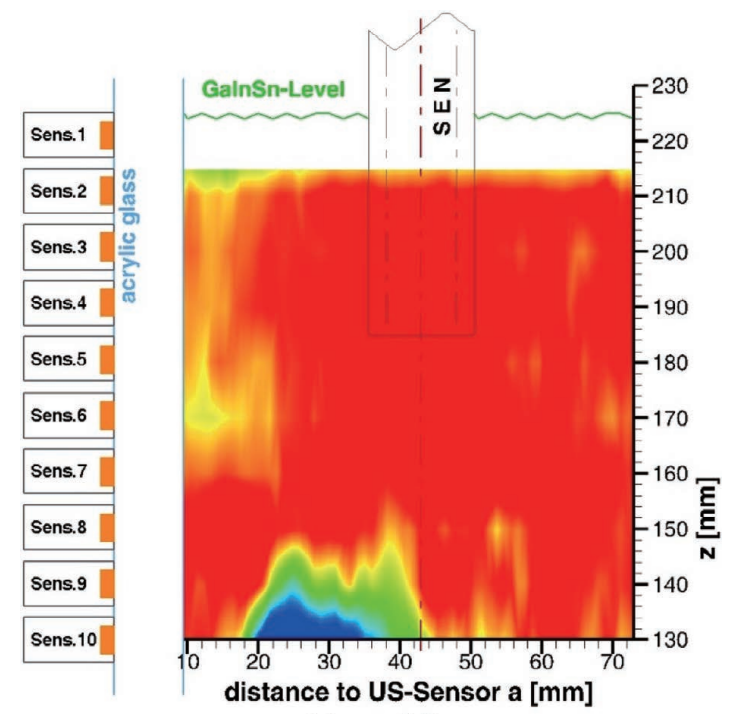

(c) $\mathrm{t}=1,9 \mathrm{~s}$

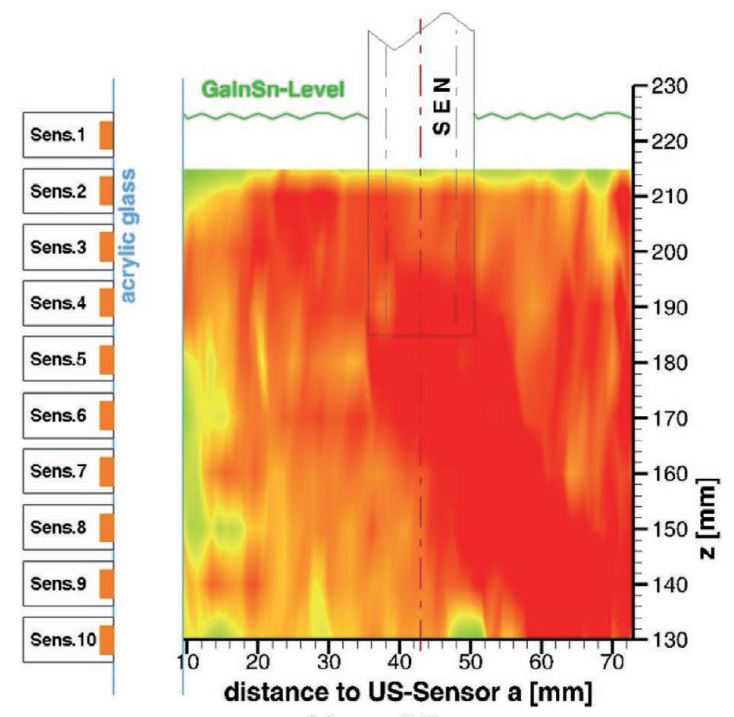

(e) $\mathrm{t}=2,8 \mathrm{~s}$

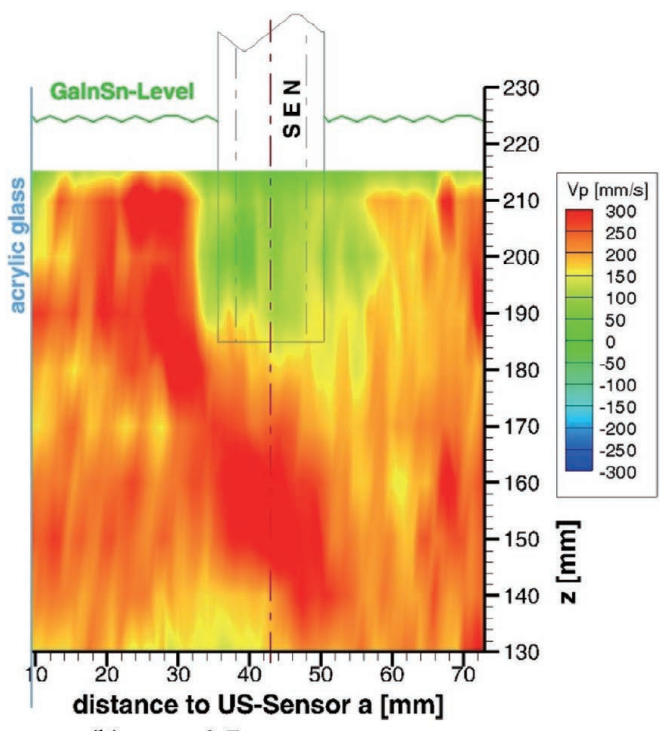

(b) $\mathrm{t}=0,7 \mathrm{~s}$

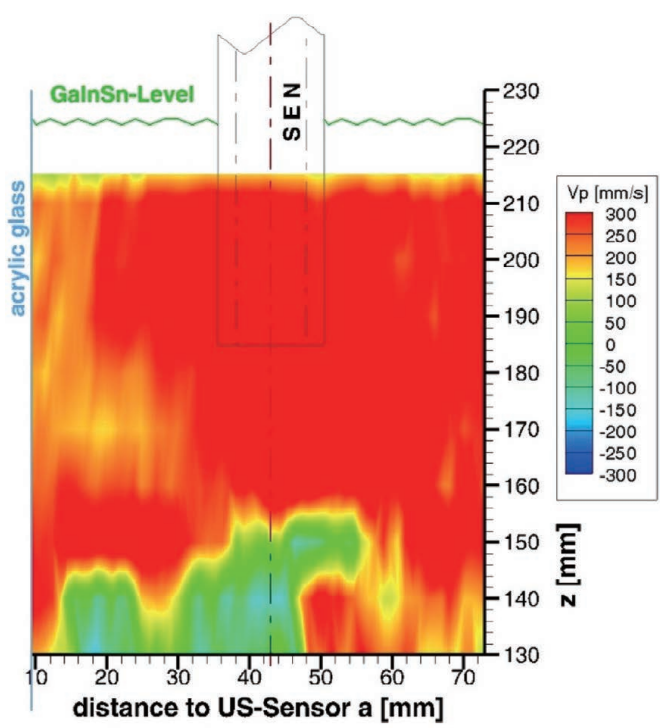

(d) $\mathrm{t}=2,0 \mathrm{~s}$

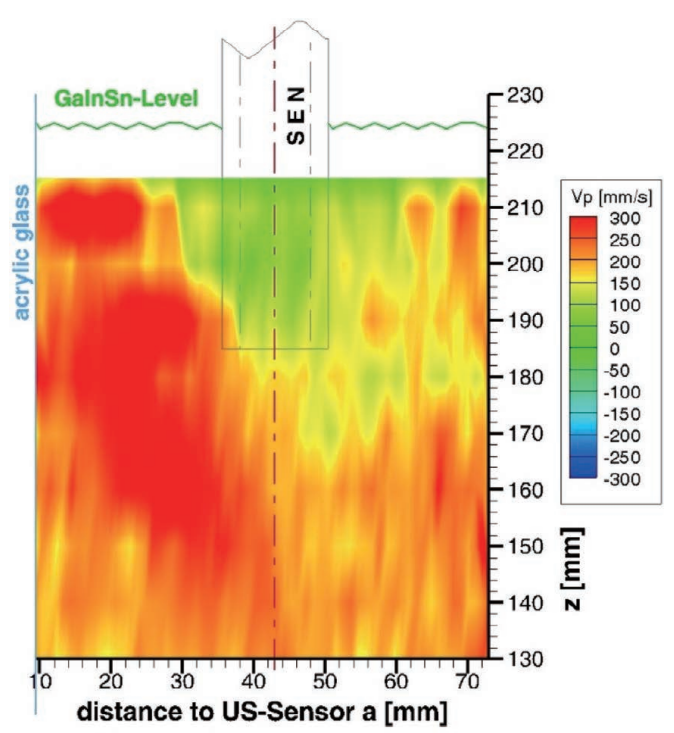

(f) $\mathrm{t}=3,4 \mathrm{~s}$

Fig. 8. Sequence of snapshots of the tangential flow recorded at $\mathrm{R}=15 \mathrm{~mm}$ within the domain $130 \mathrm{~mm}<\mathrm{z}<220 \mathrm{~mm}$. 
(10 $\mathrm{mm}$ distance between two neighbouring sensors). At a depth of $43 \mathrm{~mm}$ the ultrasound beam intersects with the radial position of $r=15 \mathrm{~mm}$. The positions of the SEN and the free surface are marked in the diagrams too. In general, the measurements reveal that the assumption of an axisymmetric flow cannot be maintained for situations with simultaneous occurrence of jet and electromagnetic stirring. For that reason the tangential flows presented here were derived from the measured values by taking the Doppler angle $\alpha$ as $\mathrm{V}_{\mathrm{p}}=\mathrm{V}_{\mathrm{US}} / \cos \alpha$ into account. Please note that the horizontal velocities are plotted here without decomposition in radial and tangential components. The radial component of the velocity was neglected here. This procedure might implicate a significant measuring error, however, the data is not taken for quantitative analysis, but rather for a qualitative identification of the dominating flow structures.

Measurements of the flow pattern were conducted for the situations of a submerged jet without swirling flow and a pure RMF-driven flow (not shown here). While no significant horizontal flow is observed for a pure jet flow, the velocity field generated by the RMF is clearly dominated by the swirling flow indicated by the gradual increase of the velocity towards larger radii. The measurements show that large-scale temporal fluctuations of the flow structure are almost negligible in both cases.

In contrast, significant changes of the flow pattern become visible when the jet interacts with electromagnetic stirring. Figure 8 contains a series of images recorded at various arbitrary moments during the respective experiment. At no time the typical flow structure of an axisymmetric rotating flow can be observed here. In general, the RMF generates a swirling flow in counterclockwise direction which is reflected by positive values (red colour) in the velocity plots. A disappearance and even an inversion of the swirling flow occurs in the nozzle region or along the bottom of the measuring range. The strong modifications of the velocity near the SEN can be attributed to the existence of a small vortex in the vicinity of the nozzle which circulates around the nozzle according to the direction of fluid rotation in the strand. Such vortices have been observed at the free surface. An example is shown in the circle of Fig. 9(c). The distinct perturbations of the flow pattern found in the lower part (see Figs. 8(c) and 8(d)) can only be explained by a distinct deformation, namely a deflecting and bending, of the jet. These temporary occurring flow reversals are responsible for the strong reduction of the time-averaged tangential velocities in the upper stirring zone as it becomes evident in the Figs. 6 and 7.

\section{Discussion}

The electromagnetic stirrer installed at the cylindrical strand generates a Lorentz force inside the liquid metal driving a rotating motion in the strand which appears to be almost axisymmetric for the case of a solely RMF-driven flow without jet. Hence, maximum values of the swirling flow are found - as supposed - in the region within the electromagnetic stirrer. On one hand, measurements of the time-averaged tangential velocity near the side walls $(\mathrm{r}=30 \mathrm{~mm}$, Fig. 4(a)) confirm this expectation. On the other hand, corresponding data recorded in the core $(\mathrm{r}=$

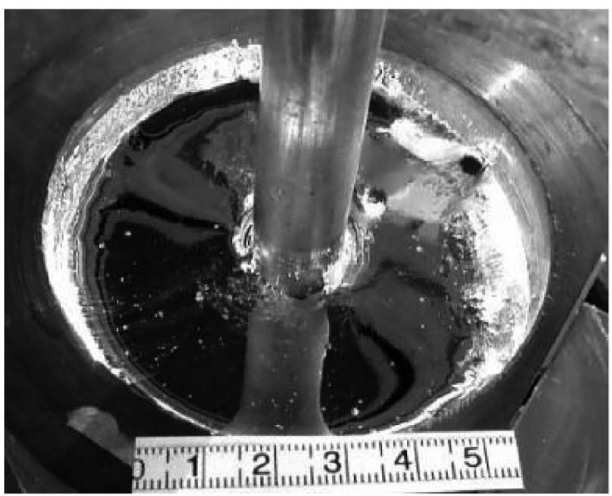

(b)

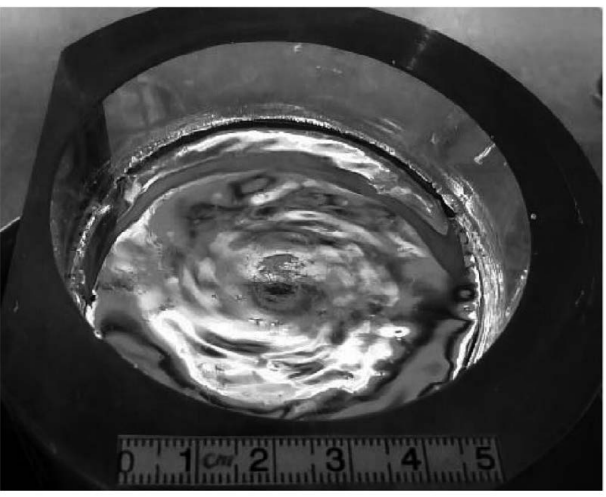

(c)

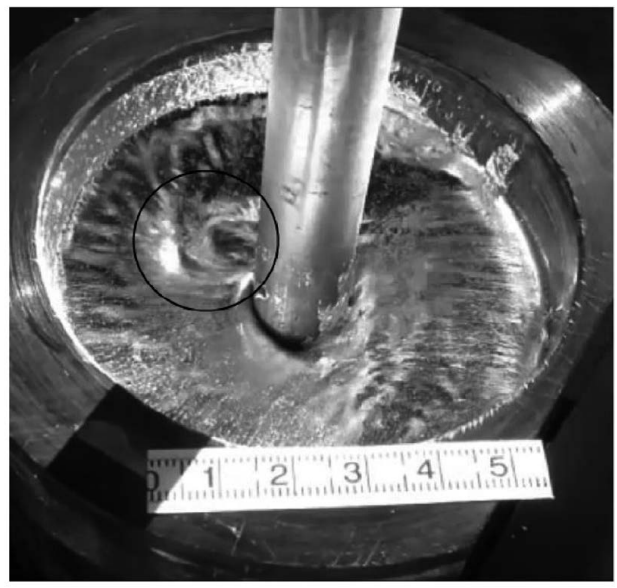

Fig. 9. Photographs of the free surface: (a) only jet; (b) only RMF, $\mathrm{B}=18.3 \mathrm{mT}, \mathrm{f}=2.5 \mathrm{~Hz}$; (c) $\mathrm{RMF}+$ jet $, \mathrm{B}=18.3 \mathrm{mT}, \mathrm{f}=$ $2.5 \mathrm{~Hz}$.

$15 \mathrm{~mm}$, Fig. 4(b)) reveal an almost uniform distribution of the tangential velocity along the height of the strand. It is interesting to note that in the region below the stirrer the core rotates faster at higher angular velocities as compared to the stirrer zone where the melt is directly affected by the electromagnetic driving force (see Fig. 4(b)). Moreover, from a comparison between Fig. 4(a) it becomes obvious that at positions far below the stirrer even higher tangential velocities are observed near the axis than at an outer radial position (e.g. $\mathrm{V}_{\mathrm{t}, \mathrm{r} 15}=115 \mathrm{~mm} / \mathrm{s}$ and $\mathrm{V}_{\mathrm{t}, \mathrm{r} 30}=87 \mathrm{~mm} / \mathrm{s}$ at a magnetic field at $\mathrm{B}=18.3 \mathrm{mT}, \mathrm{f}=2.5 \mathrm{~Hz}$ ). In their classical paper Davidson \& Hunt $^{27)}$ developed an approximate analytical model which reveals that the secondary poloidal flow plays an important role in the overall dynamics. From this model an effective depth of stirring of $z / R=2.3$ was predicted corresponding to a depth of about $90 \mathrm{~mm}$ in our case. These analytical results are explained by the conservation of angular momentum. Besides the primary rotating 
motion a secondary flow exists in the meridional plane of the strand which is directed from the sidewalls towards the cylinder axis in the regions outside the stirrer. That means that the rotating fluid is transported inwards and increases its angular velocity along this way. This mechanism is responsible for increasing tangential velocities in close vicinity to the cylinder axis and for transporting angular momentum into regions outside the stirrer.

The phenomenon of significant acceleration of the rotating fluid flow above the stirrer zone becomes more pronounced for the situation of a jet emerging from the submerged entry nozzle. In this case a striking feature of the flow field can be observed, which manifests in the occurrence of large tangential velocities up to $300 \mathrm{~mm} / \mathrm{s}$ for positions close to the free surface. These high swirling rates can be seen on the free surface where a strong vortex is formed around the SEN associated with a significant deflection of the meniscus. Obviously, the vertical jet flow amplifies the upper vortex of the secondary flow significantly because rotating fluid is conveyed from the side walls towards the SEN. The radially inwards transport of angular momentum is the reason for the distinct acceleration of the fluid near the SEN. The formation of such intensive rotating vortices has already been detected for fluid flows generated by a combination of rotating and travelling magnetic field. This swirl accumulation can even produce tornado-like flow structures in a laboratory scale. ${ }^{28,29)}$

The effect of swirl accumulation becomes visible by strong deformations of the free surface. Fig. 9 contains a comparison of photographs showing the free surface for the cases of a submerged jet without RMF (Fig. 9(a)), a rotating flow without jet (Fig. 9(b)) and the superposition of jet and RMF-driven rotating motion inside the mould. The free surface remains almost undisturbed as long as the RMF is idle. The swirling flow shows the typical parabolic shape of the melt surface, but the strongest depression of the free surface is observed directly around the nozzle in Fig. 9(c). The secondary flow which exists in every rotating flow contained in a finite vessel is significantly amplified by the impinging jet. The secondary flow driven by rotation and the jet flow have the same direction, but, the intensity of the jet flow is approximately two orders of magnitude stronger. Therefore, the acceleration of the rotation near the SEN and the deflection of the free surface are much more pronounced with the jet flow as for the case of the pure RMF-driven flow. Here, the fluid above the stirrer rotates much faster than inside the magnetically forced region, the angular velocity even exceeds the magnetic field frequency of $\mathrm{f}=2.5 \mathrm{~Hz}$. Small vortices may occur at the outer nozzle wall. In Fig. 9(c) such a vortex is marked by a black circle. It was already discussed above that this vortex revolving around the SEN with the RMF-driven flow impacts the velocity measurements as shown in Fig. 8. It appears intermittently as a green-dominated (Figs. 8(b), 8(f)) spot at heights between $220 \mathrm{~mm}$ and $180 \mathrm{~mm}$ indicating a locally vanishing rotating flow between the free surface and a height where the submerged jet enters the mould. Moreover, the flow mapping of the time-dependent tangential flow (Fig. 8) indicates a radial deflection of the jet position. At a distance of about $40 \mathrm{~mm}$ below the nozzle port the jet is clearly detected at a radial position of $15 \mathrm{~mm}$ corresponding to a deflection angle of about $22^{\circ}$. The jet position is found to vary in time, as the jet is advected by the rotating flow. Both the jet and the manifestation of the small vortex appear in the images of the tangential flow pattern with the same periodic time, but shifted for half a rotation period. This indicates an inherent coupling between these two flow structures.

Figure 10 compares the angular velocities for the jet and the rotating bulk flow measured at $\mathrm{z}=130 \mathrm{~mm}$ which is the vertical position $55 \mathrm{~mm}$ below the nozzle port. It is interesting to note that at both radial positions $(\mathrm{r}=15 \mathrm{~mm}$ and $\mathrm{r}=30 \mathrm{~mm}$ ) the purely electromagnetically driven flow rotates with less speed as compared to the combination of RMF-driven flow and submerged jet. Especially, extraordinary high angular frequencies are found at $\mathrm{r}=15 \mathrm{~mm}$ which even exceed the frequency of the RMF $(\omega=2 \pi \mathrm{f}=$ $15.7 \mathrm{~s}^{-1}$ ). The secondary flow is amplified by the jet leading to an enhanced transfer of angular momentum from the side walls towards the axis and causing therefore this considerable increase of the circulation frequencies in the core region of the upper part of the mould. It is interesting to note that a remarkable swirl enhancement can also be observed at larger radii $(\mathrm{r}=30 \mathrm{~mm})$. Obviously, a part of the kinetic energy supplied by the jet is transferred to the swirling motion in the strand. A recurring appearance of the jet has been detected by the measurements presented in Fig. 8. The temporary distinct perturbations of the flow pattern, namely the reversals of the flow direction (see Fig. 8(c)) in the lower part of the measuring domain, are a distinct footprint of the jet. The jet is deflected radially outwards and moves with swirling flow. The lower curve in Fig. 10 represents the angular frequencies of the jet which turn out to be distinctly lower as those measured for the rotating bulk flow. Likely, this difference can be explained by the high inertia of the inflowing jet which is not yet accelerated up to the mean rotation speed of the bulk flow here. The jet shows an irregular behaviour at low magnetic field strengths $(\mathrm{B}<13 \mathrm{mT})$. No dominating angular frequency of the jet can be determined in this parameter region. The reason for this behaviour cannot be clarified so far. Obviously, the interaction between the submerged jet and the swirling flow appears to be rather complex. For instance, the flow measurements presented in this paper cannot fully clarify the reason for the flow reversals shown in Fig. 8.

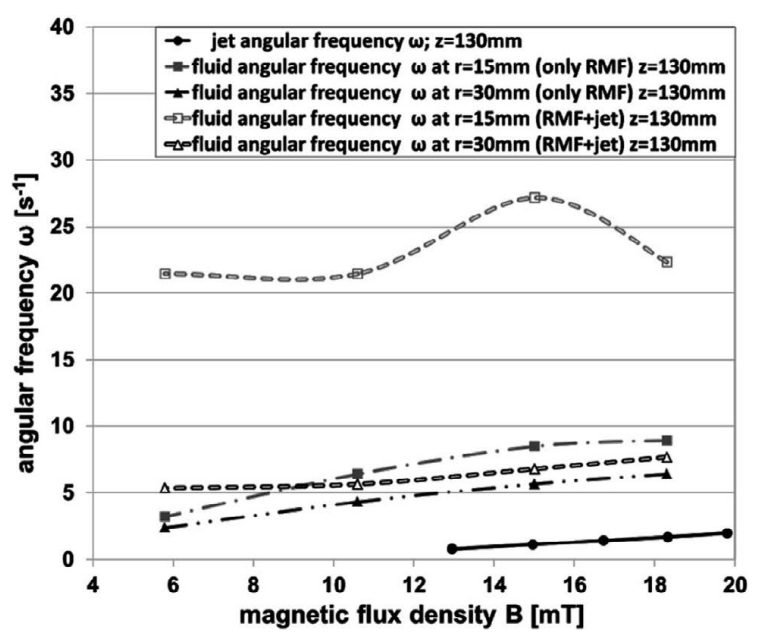

Fig. 10. Comparison of the rotation rates of the jet and the rotating liquid in the strand. 
This feature can be caused by a bending of the jet against the direction of the rotating bulk flow or it can arise from a strong intrinsic jet rotation. Further investigations will be necessary to answer these open questions.

\section{Conclusions}

This paper describes laboratory experiments in a 1:3 scale model for continuous casting of round blooms at voestalpine Stahl Donawitz GmbH. The model mould has an inner diameter of $80 \mathrm{~mm}$ and a length of $800 \mathrm{~mm}$ and was filled with the eutectic alloy GaInSn, which is liquid at room temperature. A magnetic stirring system was placed just below the SEN outlet. A rotating magnetic field was applied to drive a swirling flow in the mould. Fluid velocities inside the cylindrical mould were measured non-invasively by means of the ultrasound Doppler velocimetry (UDV). The measuring setup enables the detection of the horizontal flow being a combination of the tangential and the radial velocity component.

The flow measurements of time-averaged velocity profiles can be summarized by the following conclusions:

(1) The electromagnetically driven mould flow is composed of a primary, swirling flow and a meridional secondary flow which consists of two toroidal vortices lying on top of each other. This secondary flow is responsible for a redistribution of angular momentum within the strand.

(2) Measurements of the tangential velocity for the situation of a pure RMF-driven flow at $r=30 \mathrm{~mm}$ show a maximum rotation speed in the stirrer region and a continuous decrease of the tangential velocity with growing distance to the stirrer. On contrary, the tangential velocity does not vary significantly over the height at $\mathrm{r}=15 \mathrm{~mm}$. In fact, the tangential speed outside the stirrer is even slightly higher as in the stirrer region which can be attributed to the angular momentum transfer by the secondary flow.

(3) Off-centre measurements at $\mathrm{R}=15 \mathrm{~mm}$ with respect to the cylinder axis allow the reconstruction of the velocity distribution over the strand cross section for radii greater than $\mathrm{r}=15 \mathrm{~mm}$.

(4) A discharging jet through the SEN intensifies the meridional flow near the free surface. The superposition with the RMF-driven flow leads to a drastic acceleration of the rotating flow and a vortex formation near the SEN. This is accompanied by a strong depression of the meniscus at the nozzle. In the real casting process, such a flow phenomenon is known to carry the risk of slag entrainment.

(5) A rather complex interaction between the jet and the rotating flow in the strand can be observed. The jet revolves at a distinctly lower rotation rate as the bulk flow. Further flow measurements (for instance a simultaneous detection of all three velocity components) are supposed to deliver more information concerning the alignment and deformation of the jet flow in the deeper zones of the mould and to contribute to a better understanding of the behaviour of the jet. This is a work in progress.

Further measurements are in progress in order to obtain more detailed information concerning the alignment and behaviour of the jet flow in the deeper zones of the mould in presence of electromagnetic stirring.
Experimental setups of cold liquid metal models are an important tool for the investigation of flow and transport processes in continuous casting. Moreover, these model experiments provide a valuable data base for benchmarking or validation of numerical simulations.

\section{Acknowledgements}

The research program of the Competence Center for Excellent Technologies in "Advanced Metallurgical and Environmental Process Development" (K1-MET) has been financially supported within the Austrian competence centre programme COMET (Competence Center for Excellent Technologies) by the Federal Ministry of Economy, Family and Youth; by the Federal Ministry for Transport, Innovation and Technology; by the provinces of Upper Austria, Styria and Tyrol, by the Styrian Business Promotion Agency and by the Tiroler Zukunftsstiftung. The authors from HZDR acknowledge additional financial support from the German Helmholtz Association in the framework of the Helmholtz Alliance LIMTECH.

\section{REFERENCES}

1) O. Ilegbusi and J. Szekely: ISIJ Int., 29 (1989), 1031.

2) S. Asai: Proc. 6th Int. Iron and Steel Cong., ISIJ, Tokyo, (1990), 370.

3) K.-H. Spitzer and O. Pesteanu: Proc. 3rd Int. Symp. on Electromagnetic Processing of Materials, ISIJ, Tokyo, (2000), 409.

4) S. Kunstreich: Rev. Metall., 4 (2003), 395, 1043.

5) S. Kittaka, K. Watanabe, T. Kanki and Y. Miura: Nippon Steel Tech. Rep., 86 (2002), 68.

6) W. Yamada, A. Kiyosa, J. Nakashima, J. Fukuda, K. Okazawa and K. Miyazawa: CAMP-ISIJ, 12 (1999), 682.

7) T. Matsumiya: ISIJ Int., 46 (2006), 1800.

8) Y. Miki, H. Kitaoka, T. Sakuraya and T. Fujii: ISIJ Int., 32 (1992), 142.

9) Z.-J. Su, J. Chen, K. Nakajima and J.-C. He: Steel Res. Int., 80 (2009), 824.

10) F.-C. Chang, J. R. Hull and L. Beitelman: Metall. Mater. Trans. B, 35B (2004), 1129.

11) K.-H. Spitzer, M. Dubke and K. Schwerdtfeger: Metall. Trans. B, 17B (1986), 119

12) J. Partinen, N. Saluja, J. Szekely and J. Kirtley: ISIJ Int., 34 (1994), 707.

13) T. T. Natarajan and N. El-Kaddah: Appl. Math. Modell., 28 (2004), 47.

14) K. Okazawa, T. Toh, J. Fukuda, T. Kawase and M. Toki: ISIJ Int., 41 (2001), 851.

15) O. Pesteanu and K. Schwerdtfeger: ISIJ Int., 43 (2003), 1556.

16) M. Javurek, M. Barna, P. Gittler, K. Rockenschaub and M. Lechner: Steel Res. Int., 79 (2008), 617.

17) M. Barna: Investigations of the Liquid Steel Flow in a Round Bloom Caster with an Electromagnetic Mould-stirrer, JKU-Linz, Linz, (2013).

18) J. Partinen, J. Szekely, C. Vives and L. Holappa: ISIJ Int., 35 (1995), 292.

19) Y. Takeda: Nucl. Eng. Des., 126 (1991), 277.

20) D. Brito, H. C. Nataf, P. Cardin, J. Aubert and J. P. Masson: Exp. Fluids, 31 (2001), 653.

21) S. Eckert, A. Cramer and G. Gerbeth: MagnetohydrodynamicsHistorical Evolution and Trends, eds. by S. Molokov, R. Moreau, H. K. Moffatt, Springer, Berlin, (2007), 275.

22) S. Franke, L. Büttner, J. Czarske, D. Räbiger and S. Eckert: Flow Meas. Instrum., 21 (2010), 402.

23) K. Timmel, S. Eckert, G. Gerbeth, F. Stefani and T. Wondrak: ISIJ Int., 50 (2010), 1134.

24) K. Timmel, S. Eckert and G. Gerbeth: Metall. Mater. Trans. B, 42B (2011), 68.

25) Y. Plevachuk, V. Sklyarchuk, S. Eckert, G. Gerbeth and R. Novakovic: J. Chem. Eng. Data, 59 (2014), 757.

26) Signal Processing (SA): http://www.signal-processing.com, (accessed 2016-09-01)

27) P. A. Davidson and J. C. R. Hunt: J. Fluid Mech., 185 (1987), 67.

28) I. Grants, C. Zhang, S. Eckert and G. Gerbeth: J. Fluid Mech., 616 (2008), 135.

29) T. Vogt, I. Grants, S. Eckert and G. Gerbeth: J. Fluid Mech., 736 (2013), 641. 